Pacific Journal of Mathematic 


\title{
PROJECTIVE QUASI-COHERENT SHEAVES OF MODULES
}

\author{
RICHARD A. LEVARO
}

\begin{abstract}
Let $R$ be a commutative ring and $\tilde{R}$ the structure sheaf over the prime spectrum of $R$.

THEOREM: Suppose $R$ has only finitely many minimal primes. Then $\tilde{R}$ is a projective $\tilde{R}$-Module if and only if $R$ is a finite direct product of local rings.
\end{abstract}

Let $R$ be a nonzero commutative ring with identity, and let $x=\operatorname{Spec}(R)$, the prime spectrum of $R$ endowed with the Zariski topology. Let $\widetilde{R}$ be the structure sheaf of $R$ on $X$. We shall use the terminology and notation of [5] in describing the category of $\widetilde{R}$-Modules, $\operatorname{Mod}(\widetilde{R})$.

There is a functor $T: \bmod (R) \rightarrow \operatorname{Mod}(\widetilde{R})$ given $T(M)=\tilde{M}$ and $T(f)=\widetilde{f}$, where $\tilde{M}$ is the $\widetilde{R}$-Module associated to $M$, and $\widetilde{f}$ is defined at each stalk of $\widetilde{M}$ to be the localization of $f$. The functor $T$ is full, faithful and exact; moreover $T$ preserves direct sums [5, Corollaire I.1.3.8 and I.1.3.9.]. In addition, $T$ determines an equivalence between $\bmod (R)$ and the category of quasi-coherent $\widetilde{R}$-Modules. In $\S 1$, we shall show that if $\widetilde{R}$ is a generator, then $\operatorname{Mod}(\widetilde{R})$ is equivalent to $\bmod (R)$. In $\S 2$ necessary and sufficient conditions are given for $\widetilde{R}$ to be a projective $\widetilde{R}$-Module.

The author wishes to thank J.J. Rotman and the Referee for their suggestions regarding the preparation of this paper.

1. The equivalence of $\operatorname{Mod}(\widetilde{R})$ and $\bmod (R)$. C. J. Mulvey [8] has given a necessary and sufficient condition for $\widetilde{R}$ to be a generator in $\operatorname{Mod}(\widetilde{R})$. For the case of the affine scheme ( $X=$ $\operatorname{Spec}(R), \widetilde{R})$, we can state Mulvey's condition as follows:

Proposition 1.1 (Mulvey, [8]). A necessary and sufficient condition that $\widetilde{R}$ be a generator in $\operatorname{Mod}(\widetilde{R})$ is that the stalks of $\widetilde{R}$ may be generated by global sections of $\widetilde{R}$ of arbitrarily small support. If this condition holds, then $X=\operatorname{Spec}(R)$ is necessarily a regular topological space.

THEOREM 1.2. The following are equivalent:

(i) $T: \bmod (R) \rightarrow \operatorname{Mod}(\widetilde{R})$ is an equivalence of categories, i.e., every $\widetilde{R}$-Module is quasi-coherent;

(ii) $\widetilde{R}$ is a generator for the category $\operatorname{Mod}(\widetilde{R})$;

(iii) $X=\operatorname{Spec}(R)$ is $T_{1}$;

(ivi) $R / N(R)$ is von Neumann regular, where $N(R)$ is the nil- 
radical of $R$. If $\widetilde{R}$ is a flabby (flasque) $\widetilde{R}$-Module, then the equivalent conditions (i)-(iv) are satisfied.

Proof. (i) implies (ii). Since $R$ is a generator of $\bmod (R)$, this implication is clear.

(ii) implies (i). Since $\widetilde{R}$ is a generator, it is immediate that every $\widetilde{R}$-Module is of the form $\tilde{M}$.

(ii) implies (iii). Because $\widetilde{R}$ is a generator, by Proposition 1.1, $X=\operatorname{Spec}(R)$ is a regular topological space. But $X$ is always $T_{0}$, so it is also $T_{1}$.

(iii) implies (iv). This is well-known and appears as a exercise in [2, page 143].

(iv) implies (ii). Since $R / N(R)$ is von Neumann regular and $X=$ $\operatorname{Spec}(R)$ is homeomorphic to $\operatorname{Spec}(R / N(R)), X$ has a basis of closed and open sets. We shall use the criterion of Proposition 1.1 to show $\widetilde{R}$ is a generator. Let $x \in X$, and let $U$ be an open set in $X$ with $x \in U$. Let $V$ be an open and closed (basic) set such that $x \in V \subseteq U$. Define sections $s_{1} \in \widetilde{R}(V)$ and $s_{0} \in \widetilde{R}(X-V)$ by $s_{1}(z)=1_{z} \in R_{p_{z}}$ for all $z \in V$, and $s_{0}(z)=0_{z} \in R_{p_{z}}$ for all $z \in X-V$. Since $V$ partitions $X$, we can collate $s_{1}$ and $s_{0}$ to obtain a global section $s$ of $\widetilde{R}$ with $s(z)=1_{z}$ if $z \in V$ and $s(z)=0_{z}$ if $z \notin V$. Clearly $s$ generates $\widetilde{R}_{x}$, and the support of $s$ is $V \cong U$. Therefore, by the Proposition, $\widetilde{R}$ is a generator.

For the last statement, suppose $\widetilde{R}$ is flabby and $s \in R$. Then the restriction map $\widetilde{R}(X) \rightarrow \widetilde{R}(D(s)$ ) is onto, and hence the localization map $R \rightarrow R_{s}$ is onto. Now $D(s) \approx \operatorname{Spec}\left(R_{s}\right)$, and because $R \rightarrow R_{s}$ is onto, Spec $\left(R_{s}\right)$ is a homeomorphic to a closed set of $X$. Hence the usual basis is both open and closed; therefore points in $X$ are closed and $X$ is $T_{1}$.

R. Wiegand has shown, using different techniques, that a reduced prescheme $(X, \mathscr{R})$ is regular (i.e., $X$ can be covered by open sets $U_{i}$ such that $\left(U_{i}, \mathscr{R} \mid U_{i}\right)$ is the affine scheme of a von Nuemann regular ring) if and only if every $\widetilde{R}$-Module is quasi-coherent [9].

The Theorem provides examples of rings for which there are projectives in $\operatorname{Mod}(\widetilde{R})$.

CoRollary 1.3. Suppose $R / N(R)$ is von Neumann regular where $N(R)$ is the nilradical of $R$. The $\widetilde{R}$-Module $\boldsymbol{F}$ is projective if and only if $\boldsymbol{F}(X)$ is a projective $R$-module. In particular, $P$ is a projective $R$-module if and only if $\widetilde{P}$ is a projective $\widetilde{R}$-Module.

2. Projective quasi-coherent $\widetilde{R}$-Modules. Suppose $\widetilde{R}$ is a projective $\widetilde{R}$-Module. If $P$ is a projective $R$-module, then there is an $R$-module $Q$ such that $P \oplus Q \cong \sum R$; hence $\widetilde{P} \oplus \widetilde{Q} \cong \sum \widetilde{R}$ since $T$ 
preserves direct sums. Therefore, $\widetilde{P}$ is a projective $\widetilde{R}$-Module. Thus, to discover when projective $R$-module yield projective $\widetilde{R}$-Modules, it is enough to determine when $\widetilde{R}$ is projective.

Proposition 2.1. If $R$ is a local (not necessarily Noetherian) ring, then $\widetilde{R}$ is a projective $\widetilde{R}$-Module.

Proof. Since $\operatorname{Hom}_{\tilde{R}}(\widetilde{R}, F)$ is naturally isomorphic to $\boldsymbol{F}(X)$ for every $\widetilde{R}$-Module $F$, we need only show the global section functor is exact. Let $p_{x}$ be the unique maximal ideal of $R$. For any $\widetilde{R}$-Module $\boldsymbol{F}, \boldsymbol{F}_{x}=\lim \boldsymbol{F}(U)$ where the direct limit is taken over all open sets contaiinng $x$. Because $X=\operatorname{Spec}(R)$ is the only open set containing $x, F_{x}=F(X)$. Now, the formation of stalks is exact, so $\operatorname{Hom}_{\tilde{R}}(\widetilde{R}$, is exact, i.e., $\widetilde{R}$ is projective.

R. Bkouche [1] introduced the notion of soft rings.

Definition. The ring $R$ is soft (mou) if $\operatorname{Max}(R)$, the maximal spectrum of $R$, is Hausdorff and $J(R)=0$, where $J(R)$ is the Jacobson radical of $R$.

For our purposes, we need a notion a bit more general.

Definition. The ring $R$ is quasi-soft if for every $x \in \operatorname{Max}(R)$, the localization map $\alpha_{x}: R \rightarrow R_{p_{x}}$ is onto.

Every local ring is quasi-soft, but not necessarily soft. Every von Neumann regular ring is quasi-soft. The relation between soft and quasi-soft rings is given by the following.

Proposition 2.2. If $R$ is quasi-soft, then $R / J(R)$ is soft, where $J(R)$ is the Jacobson radical of $R$. Every soft ring is quasi-soft.

Proof. If $R$ is quasi-soft, then $\operatorname{Max}(R)$ is regular as can be seen by imitating the proof for soft rings [1, Proposition 1.6.1 and 1.6.2]. But $\operatorname{Max}(R)$ is always $T_{1}$; hence $\operatorname{Max}(R)$ is Hausdorff. Since $\operatorname{Max}(R) \approx \operatorname{Max}(R / J(R))$ and $J(R / J(R))=0, R / J(R)$ is soft.

Now suppose $R$ is soft, $x \in \operatorname{Max}(R)$, and let $\alpha_{x}: R \rightarrow R_{p_{x}}$ be the localization map. Because $J(R)=0$ and $\operatorname{Max}(R)$ is Hausdorff, $V_{M}\left(\operatorname{ker}\left(\alpha_{x}\right)\right)=\{x\}$, where $V_{M}(I)=\operatorname{Max}(R) \cap V(I)$ for an ideal $I$ of $R$. Therefore, $R / \operatorname{ker}\left(\alpha_{x}\right)$ is a local ring with maximal ideal $p_{x}$, and so every element outside $p_{x}$ is invertible. By the universal mapping property of localization, $R / \operatorname{ker}\left(\alpha_{x}\right) \cong R_{p_{x}}$; hence $R$ is quasi-soft. 
Quasi-softness is the condition we must investigate to find necessary conditions for $\widetilde{R}$ to be a projective $\widetilde{R}$-Module in view of the following result.

Proposition 2.3. If $\widetilde{R}$ is a projective $\widetilde{R}$-Module, then $R$ is quasi-soft.

Proof. Let $x \in \operatorname{Max}(R)$ and set $A=\{x\}$. Then $A \subseteq X$ is closed, and we have the exact sequence

$$
0 \longrightarrow \widetilde{R}_{X-4} \longrightarrow \widetilde{R} \stackrel{\alpha}{\longrightarrow} \widetilde{R}_{A} \longrightarrow 0
$$

of $\widetilde{R}$-Modules [4, Théorème 2.9.3.]. Since $\widetilde{R}$ is projective, $\operatorname{Hom}_{\tilde{R}}(\widetilde{R}$, is exact, and hence $\operatorname{Hom}_{\widetilde{R}}(\widetilde{R}, \widetilde{R}) \stackrel{\alpha_{*}}{\rightarrow} \operatorname{Hom}_{\widetilde{R}}\left(\widetilde{R}, \widetilde{R}_{A}\right)$ is onto. Now $\operatorname{Hom}_{\widetilde{R}}(\widetilde{R}, \widetilde{R}) \cong R$ and $\operatorname{Hom}_{\widetilde{R}}\left(\widetilde{R}, \widetilde{R}_{A}\right) \cong R_{p_{x}}$, and it is routine to check that $\alpha_{*}$ may be identified with the localization map $\alpha_{x}: R \rightarrow R_{p_{x}}$ (i.e., the obvious diagram commutes). Therefore $R$ is quasi-soft.

We can now state and prove the

MAIN THEOREM. Suppose $R$ has only finitely many minimal primes. Then $\widetilde{R}$ is a projective $\widetilde{R}$-Module if and only if $R$ is finite direct product of local rings.

Proof. Since $R$ has only finitely many minimal primes, $R$ is the finite direct product of connected rings, say $R=R_{1} \times R_{2} \times \cdots \times R_{n}$ each having only finitely many minimal primes. If $\widetilde{R}$ is a projective $\widetilde{R}$-Module, $\widetilde{R}_{i}$ is a projective $\widetilde{R}_{i}$-module for each $i$. By Proposition $2.3 R_{i}$ is quasi-soft. Hence $\operatorname{Max}\left(R_{i}\right)$ is finite, since each prime ideal of a quasi-soft, ring is contained in a unique maximal ideal [1, Proposition 1.6.1]. Also, since $R_{i}$ is quasi-soft, $\operatorname{Max}\left(R_{i}\right)$ is the continuous image of $\operatorname{Spec}\left(R_{i}\right)$ [1, Proposition 1.6.2]. (See also [3]). Thus, $\operatorname{Max}\left(R_{i}\right)$ is finite and discrete, but also connected being the continuous image of $\operatorname{Spec}\left(R_{i}\right)$. Therefore $\operatorname{Max}\left(R_{i}\right)$ consists of a single point, and hence $R_{i}$ is local.

Conversely, if $R=R_{1} \times \cdots \times R_{n}$ where each $R_{i}$ is local, then $\widetilde{R}_{i}$ is a projective $\widetilde{R}_{i}$-Module by Proposition 2.1. Hence, $\widetilde{R}$ is a projective $\widetilde{R}$-Module.

The Main Theorem resolves the problem of determining the projectivity of $\widetilde{R}$ for rings with only finitely many minimal primes; in particular, for Noetherian rings and integral domains.

Let $R$ be a discrete valuation domain. In this case; $X=\operatorname{Spec}(R)=$ $\{(0), p\}$, where $p$ is the unique maximal ideal of $R$. Since $R$ is local, 
$\widetilde{R}$ is a projective $\widetilde{R}$-Module. Since $U=\{(0)\}$ is smallest open set containing $(0), \widetilde{R}_{U}$ is also a projective $\widetilde{R}$-Module. Thus, there are examples of projective $\widetilde{R}$-Modules which are not quasi-coherent. Furthermore, since $\widetilde{R} \oplus \widetilde{R}_{U}$ is a generator for $\operatorname{Mod}(\widetilde{R})$ [6, Proposition 3.1.1], in this case $\operatorname{Mod}(\widetilde{R})$ has a small projective generator. Hence $\operatorname{Mod}(\widetilde{R})$ is equivalent to a category of modules [7, Theorem 4.1, page 104], but the functor $T$ is not the equivalence since $X=$ $\operatorname{Spec}(R)$ is not $T_{1}$.

\section{REFERENCES}

1. R. Bkouche, Couples spectraux et faisceaux associés. Applications aux anneaux fonctions, Bull. Soc. Math. France, 98 (1970), 253-295.

2. N. Bourbaki, Elements of Mathematics, Commutative Algebra, Addison-Wesley, Reading, Massachusetts, 1972.

3. G. De Marco and A. Orsatti, Commutative rings in which every prime ideal is contained in a unique maximal ideal, Proc. Amer. Math. Soc., 30 (1971), 459-466.

4. R. Godement, Topological Albébrique et Théorie des Faisceaux, Hermann, Paris, 1958.

5. A. Grothendieck and J. A. Dieudonné, Eléments de Géométrie Algébrique I, SpringerVerlag, Berlin, 1972.

6. A. Grothendieck, Sur quelques points d'algèbre homologique, Tôhoku Math. J., 9 (1957), 119-221.

7. B. Mitchell, Theory of Categories, Academic Press, New York and London, 1965.

8. C. J. Mulvey, $A$ condition for a ringed space to be a generator in its category of modules, J. Algebra, 15 (1970), 312-313.

9. R. Wiegand, Generators of modules over commutative rings, J. Algebra, 27 (1973), 454-461.

Received November 11, 1974 and in revised form March 10, 1975.

WASHINGTON UNIVERSITY 



\section{PACIFIC JOURNAL OF MATHEMATICS}

\section{EDITORS}

RICHARD ARENS (Managing Editor)

University of California

Los Angeles, California 90024

\section{J. DugundJI}

Department of Mathematics University of Southern California Los Angeles, California 90007

D. Gilbarg and J. Milgram

Stanford University

Stanford, California 94305
University of Washington Seattle, Washington 98105

\section{ASSOCIATE EDITORS}
E. F. BECKENBACH
B. H. NeumanN
F. WolF
K. YoShIDA

\section{SUPPORTING INSTITUTIONS}

\author{
UNIVERSITY OF SOUTHERN CALIFORNIA \\ STANFORD UNIVERSITY \\ UNIVERSITY OF TOKYO \\ UNIVERSITY OF UTAH \\ WASHINGTON STATE UNIVERSITY \\ UNIVERSITY OF WASHINGTON \\ $\stackrel{*}{*} \stackrel{*}{*} \stackrel{*}{ }{ }^{*}$ AMERICAN MATHEMATICAL SOCIETY
}

The Supporting Institutions listed above contribute to the cost of publication of this Journal, but they are not owners or publishers and have no responsibility for its content or policies.

Mathematical papers intended for publication in the Pacific Journal of Mathematics should be in typed form or offset-reproduced, (not dittoed), double spaced with large margins. Underline Greek letters in red, German in green, and script in blue. The first paragraph or two must be capable of being used separately as a synopsis of the entire paper. Items of the bibliography should not be cited there unless absolutely necessary, in which case they must be identified by author and Journal, rather than by item number. Manuscripts, in triplicate, may be sent to any one of the editors. Please classify according to the scheme of Math. Reviews, Index to Vol. 39. All other communications should be addressed to the managing editor, or Elaine Barth, University of California, Los Angeles, California, 90024.

The Pacific Journal of Mathematics expects the author's institution to pay page charges, and reserves the right to delay publication for nonpayment of charges in case of financial emergency.

100 reprints are provided free for each article, only if page charges have been substantially paid. Additional copies may be obtained at cost in multiples of 50 .

The Pacific Journal of Mathematics is issued monthly as of January 1966. Regular subscription rate: $\$ 72.00$ a year (6 Vols., 12 issues). Special rate: $\$ 36.00$ a year to individual members of supporting institutions.

Subscriptions, orders for back numbers, and changes of address should be sent to Pacific Journal of Mathematics, 103 Highland Boulevard, Berkeley, California, 94708.

\section{PUBLISHED BY PACIFIC JOURNAL OF MATHEMATICS, A NON-PROFIT CORPORATION}

Printed at Kokusai Bunken Insatsusha (International Academic Printing Co., Ltd.), 270, 3-chome Totsuka-cho, Shinjuku-ku, Tokyo 160, Japan.

\section{Copyright (C) 1975 by Pacific Journal of Mathematics} Manufactured and first issued in Japan 


\section{Pacific Journal of Mathematics}

\section{Vol. 57, No. $2 \quad$ February, 1975}

Norman Larrabee Alling, On Cauchy's theorem for real algebraic curves with boundary .......

Daniel D. Anderson, A remark on the lattice of ideals of a Prüfer domain ..................

Dennis Neal Barr and Peter D. Miletta, A necessary and sufficient condition for uniqueness of

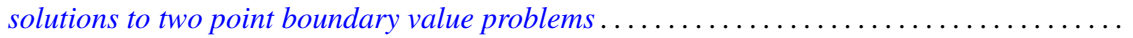

Ladislav Beran, On solvability of generalized orthomodular lattices . . . . . . . . . . ........

L. Carlitz, A three-term relation for some sums related to Dedekind sums . . . . . . . . . .....

Arthur Herbert Copeland, Jr. and Albert Oscar Shar, Images and pre-images of localization

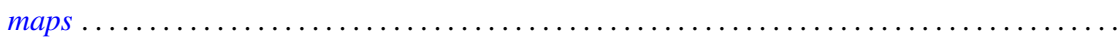

G. G. Dandapat, John L. Hunsucker and Carl Pomerance, Some new results on odd perfect

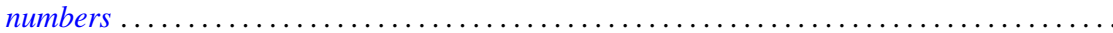

M. Edelstein and L. Keener, Characterizations of infinite-dimensional and nonreflexive

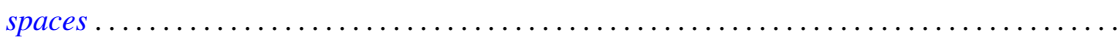

Francis James Flanigan, On Levi factors of derivation algebras and the radical embedding

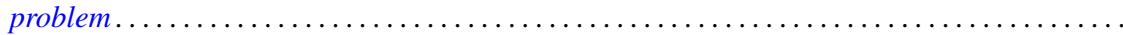

Harvey Friedman, Provable equality in primitive recursive arithmetic with and without

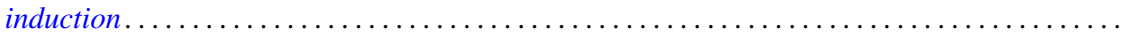

Joseph Braucher Fugate and Lee K. Mohler, The fixed point property for tree-like continua with

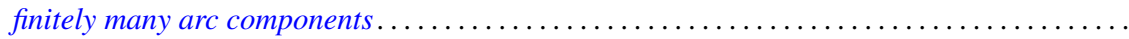

John Norman Ginsburg and Victor Harold Saks, Some applications of ultrafilters in

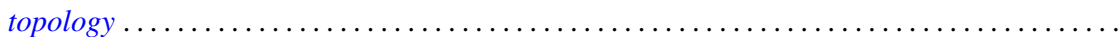

Arjun K. Gupta, Generalisation of a "square" functional equation .....................

Thomas Lee Hayden and Frank Jones Massey, Nonlinear holomorphic semigroups ..........

V. Kannan and Thekkedath Thrivikraman, Lattices of Hausdorff compactifications of a locally

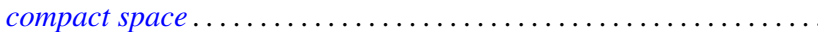

J. E. Kerlin and Wilfred Dennis Pepe, Norm decreasing homomorphisms between group

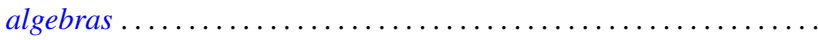

Young K. Kwon, Behavior of $\Phi$-bounded harmonic functions at the Wiener boundary ...

Richard Arthur Levaro, Projective quasi-coherent sheaves of modules .

Chung Lin, Rearranging Fourier transforms on groups...........................

David Lowell Lovelady, An asymptotic analysis of an odd order linear differential equation . . 4475

Jerry Malzan, On groups with a single involution .......................... 481

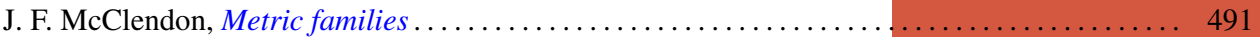

Carl Pomerance, On multiply perfect numbers with a special property .

Mohan S. Putcha and Adil Mohamed Yaqub, Polynomial constraints for finiteness of semisimple rings. .

Calvin R. Putnam, Hyponormal contractions and strong power convergence . . . . . . . . . 531

Douglas Conner Ravenel, Multiplicative operations in $\mathrm{BP} * \mathrm{BP} \ldots \ldots \ldots \ldots \ldots \ldots \ldots \ldots \ldots .539$

Judith Roitman, Attaining the spread at cardinals which are not strong limits . . . . . . . . . 545

Kazuyuki Saitô, Groups of *-automorphisms and invariant maps of von Neumann algebras . . . 553

Brian Kirkwood Schmidt, Homotopy invariance of contravariant functors acting on smooth

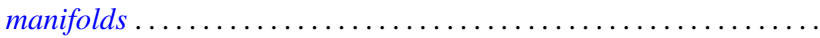

Kenneth Barry Stolarsky, The sum of the distances to $N$ points on a sphere.

Mark Lawrence Teply, Semiprime rings with the singular splitting property.

J. Pelham Thomas, Maximal connected Hausdorff spaces..............

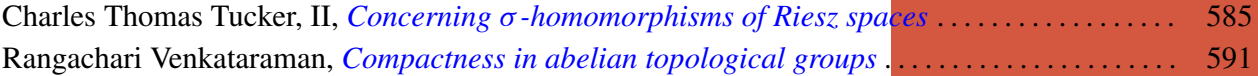

William Charles Waterhouse, Basically bounded functors and flat sheaves . . . . . . . . . . . 597

David Westreich, Bifurcation of operator equations with unbounded linearized part ......... 611

William Robin Zame, Extendibility, boundedness and sequential convergence in spaces of 